\title{
Ecotecnias para el tratamiento de residuos: experiencia de la subcuenca del río Jabonal, Costa Rica
}

\author{
Waste Treatment Echotecnics: Jabonal River \\ Sub-basin Experience, Costa Rica
}

Ecotécnicas para o tratamento de resíduos: Experiência do rio Jabonal, Costa Rica

\author{
María José Chassoul-Acosta \\ Universidad de Costa Rica, Sede Occidente. San Ramón, Costa Rica \\ maria.chassoul@ucr.ac.cr \\ https://orcid.org/0000-0002-3319-1427
}

DOI: https://doi.org/10.32719/25506641.2021.10.5

Recibido: 14 de octubre de 2020 - Revisado: 18 de enero de 2021

Aceptado: 25 de enero de 2021 • Publicado: 1 de julio de 2021

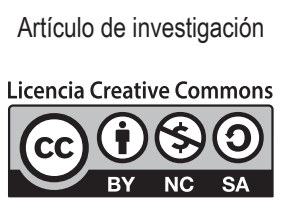




\section{Resumen}

Los resultados mostrados en este artículo son parte de la aplicación del modelo de desarrollo rural endógeno sostenible (MDRES), enfocado en el manejo de residuos por medio de ecotecnologías y la generación de actividades productivas. Este modelo se diseñó como una alternativa de solución a los problemas económicos, sociales y ambientales presentes en comunidades rurales de Costa Rica; el desarrollo rural endógeno sostenible se visualiza como un proceso emprendedor e innovador que le permite a la comunidad local mejorar su condición de vida mediante el establecimiento de actividades económicas y socioculturales utilizando básicamente sus propios recursos, pero con la apertura necesaria para modificar e integrar conocimientos externos. La mejora de la condición de vida se consigue mediante la utilización de las capacidades que han desarrollado los habitantes o por la obtención de nuevas competencias, que les permita utilizar los recursos disponibles en la zona. En el caso aquí descrito, se evidencia cómo el uso de ecotecnologías contribuye a disminuir el impacto negativo que generan las acciones de los habitantes en el ambiente y a la vez permite comprobar que desde las viviendas y las fincas se pueden desarrollar proyectos ambiental y económicamente viables.

Palabras clave: ecotecnias, tratamiento de residuos, competencias, calidad de vida, desarrollo rural.

JEL: Q55 Innovación tecnológica.

\section{Abstract}

The results in this article are part of the sustainable endogenous rural development model application (DRES), focused on waste management through eco-technologies and productive activities generation, specifically, data on eco-technologies implementation. This model was designed as an alternative solution to the economic, social and environmental problems in rural communities in Costa Rica; here, endogenous sustainable rural development is an entrepreneurial and innovative process allowing the local community to improve their living conditions by establishing economic and socio-cultural activities using basically their own resources, and willing to modify and integrate external knowledge. The improvement of the living conditions is achieved by using skills that the inhabitants have developed or obtaining new ones that allow them to use the available resources in the area. In this case the use of eco-technologies contributes to reducing the negative impact of the inhabitants on the environment, and at the same time allows us to verify weather environmentally and economically viable projects can be developed from homes and farms.

Keywords: ecotechnics, waste treatment, empowerment, quality of life, rural development. JEL: Q55 Technological innovation. 


\section{Resumo}

Os resultados apresentados neste artigo são parte da aplicação do modelo de desenvolvimento rural endógeno sustentável (DRES), enfocado no manejo de resíduos por meio de ecotecnologias e geração de atividades produtivas. Particularmente, mostram-se dados a respeito da implementação de tais ecotecnologias. Esse modelo foi formulado como uma alternativa de solução aos problemas econômicos, sociais e ambientais presentes em comunidades rurais da Costa Rica; em tal modelo, o desenvolvimento rural endógeno sustentável é visto como um processo empreendedor e inovador que permite à comunidade local melhorar sua condição de vida por meio do estabelecimento de atividades econômicas e socioculturais utilizando, basicamente, recursos próprios, mas com a abertura necessária para modificar e integrar conhecimentos externos. A melhoria das condições de vida é obtida mediante a utilização das capacidades desenvolvidas pelos habitantes ou pela obtenção de novas competências que lhes permitam utilizar os recursos disponíveis na região. No caso aqui descrito, envidencia-se como o uso de ecotecnologias contribui para diminuir o impacto negativo que geram as ações dos habitantes sobre o ambiente e, ao mesmo tempo, é possível comprovar que, a partir dos lares e das propriedades, podemse desenvolver projetos ambiental e economicamente viáveis.

Palavras-chave: ecotécnicas, tratamento de resíduos, competências, qualidade de vida, desenvolvimento rural.

JEL: Q55 Inovação tecnológica.

\section{Introducción}

L

as comunidades de Cerrillos, Sabana Bonita y Peñas Blancas se localizan en la subcuenca del río Jabonal de Esparza, Puntarenas, Costa Rica. Cuentan con una población estimada de 227 habitantes, quienes se dedican principalmente a la actividad ganadera, tanto de leche como de carne. Aproximadamente el $42 \%$ del suelo de la subcuenca se utiliza para fines agropecuarios, principalmente ganadería, el 33\% y el 58\% restante posee cobertura boscosa. Los habitantes son productores, en su mayoría, de subsistencia (Chassoul 2015).

En estos poblados, como en la mayoría de las comunidades rurales de Costa Rica, el manejo de los residuos sólidos y líquidos está a cargo de las personas que los generan. Debido a esto, del total de residuos, el $73 \%$ son quemados, el $24 \%$ se entierran y el $3 \%$ es dispuesto en lotes baldíos. También existe un inadecuado manejo de las aguas residuales de tipo ordinario generadas en las viviendas, así como de las aguas residuales de tipo especial 
provenientes de las queseras (vertidas directamente al terreno o a la quebrada); además, los residuos generados por los animales quedan en los potreros o son vertidos en la quebrada más cercana (Chassoul 2015).

Con la finalidad de ayudar en este y otros problemas presentes en dichos poblados, se diseñó el modelo de desarrollo rural endógeno sostenible (MDRES), enfocado en el manejo de residuos por medio de ecotecnologías y la generación de actividades productivas (Chassoul y Charpentier 2017). Entendiéndose como ecotecnia a los "instrumentos desarrollados para aprovechar eficientemente los recursos naturales y materiales, permitiendo la elaboración de productos y servicios, así como el aprovechamiento sostenible de los recursos naturales y materiales diversos para la vida diaria" (CDI 2016, 4).

Algunas ecotecnias identificadas son los biodigestores, las biojardineras, la lombricultura, el compost, la hidroponía y la captación de agua de lluvia. La ventaja de estas tecnologías es que se pueden elegir aquellas que sirvan para solventar las necesidades presentes en las comunidades, que se adapten al entorno y a las costumbres y que utilicen los materiales disponibles en la zona. Por ejemplo, los biodigestores son sistemas diseñados para optimizar la producción de biogás por medio de desechos orgánicos, lo cual permite obtener energía limpia, renovable y de bajo costo (Rivas, Faith y Guillén 2009). El biogás puede emplearse para cocción, iluminación y calefacción o, como demuestran Venegas et al. (2017), puede ser quemado en una turbina de gas o motor de combustión interna para generar electricidad y calor y se encuentra compuesto principalmente por metano $\left(\mathrm{CH}_{4}\right)$, dióxido de carbono $\left(\mathrm{CO}_{2}\right)$, otros gases en pequeñas concentraciones y vapor de agua (CABAL 2014). El material saliente, que queda después de producir el gas, es un fertilizante llamado biol (mezcla fangosa entre líquida y sólida), el cual sirve como abono orgánico y permite sustituir parcial o totalmente el uso de abonos químicos. Es rico en nitrógeno, fósforo y potasio (Valdez 2016).

Las biojardineras son una alternativa - con muy buenos resultadospara el tratamiento de aguas residuales provenientes de diferentes orígenes, como son las industrias, minas, casas y fincas (Brix y Arias 2006). En esta interactúan los componentes biológicos de las aguas residuales con las raíces de las plantas y la piedra haciendo que el sistema purifique el agua (Cubillo y Gómez 2017). En general, pueden tratar con eficiencia niveles altos, superiores al $80 \%$, de demanda bioquímica de oxígeno (DBO), demanda química 
de oxígeno (DQO), sólidos suspendidos totales (SST) y nitrógeno, así como niveles significativos de metales, trazas orgánicas y patógenos (UNI 2004; EPA 1993; Zhang, Gersberg y Keat 2009). Una vez que el agua contenga menos contaminantes, se puede reutilizar en el riego de jardines, patios y en usos agropecuarios, entre otros. También se puede infiltrar por medio de un drenaje o descargando en algún curso natural que exista en las cercanías.

La lombricultura es una forma de producir fertilizante orgánico o humus, realizado con la ayuda de la lombriz roja californiana. El humus es el producto resultante de la transformación digestiva en forma de excretas que realizan las lombrices sobre la materia orgánica que consume. Como abono orgánico tiene un excelente valor en nutrientes.

El objetivo de este trabajo es mostrar el resultado obtenido de la implementación del apartado de ecotecnologías del MDRES en viviendas de tres pequeñas comunidades ubicadas en la subcuenca del río Jabonal, como son Sabana Bonita, Cerrillos y Peñas Blancas, entre 2012 y 2015. La construcción de las ecotecnologías fue voluntaria y con la ayuda de la Asociación Pro Cuenca Río Jabonal y diversos proyectos de investigación y acción social de la Sede de Occidente de la Universidad de Costa Rica.

\section{Materiales y métodos}

\section{Descripción del área de estudio}

La zona presenta una precipitación media de $3200 \mathrm{~mm}$ por año, una temperatura anual media de $22{ }^{\circ} \mathrm{C}$ y una humedad relativa del $90 \%$ (Ríos et al. 2006). Tiene dos épocas bien marcadas: la lluviosa, de mayo a diciembre y la seca, de enero a abril. El $42 \%$ del suelo se utiliza para fines agropecuarios y el $58 \%$ posee cobertura boscosa.

La subcuenca está compuesta por los poblados de San Jerónimo, Cerrillos, Sabana Bonita, Peñas Blancas, Zapotal y Carrera Buena. De estos, cuatro poblados pertenecen al municipio de Esparza, Puntarenas y dos al de San Ramón, Alajuela. En la figura 1, se muestra la ubicación de la subcuenca y específicamente los poblados de Cerrillos, Sabana Bonita y Peñas Blancas. 
Figura 1

Ubicación de la subcuenca del río Jabonal y los poblados de Cerrillos, Sabana Bonita y Peñas Blancas

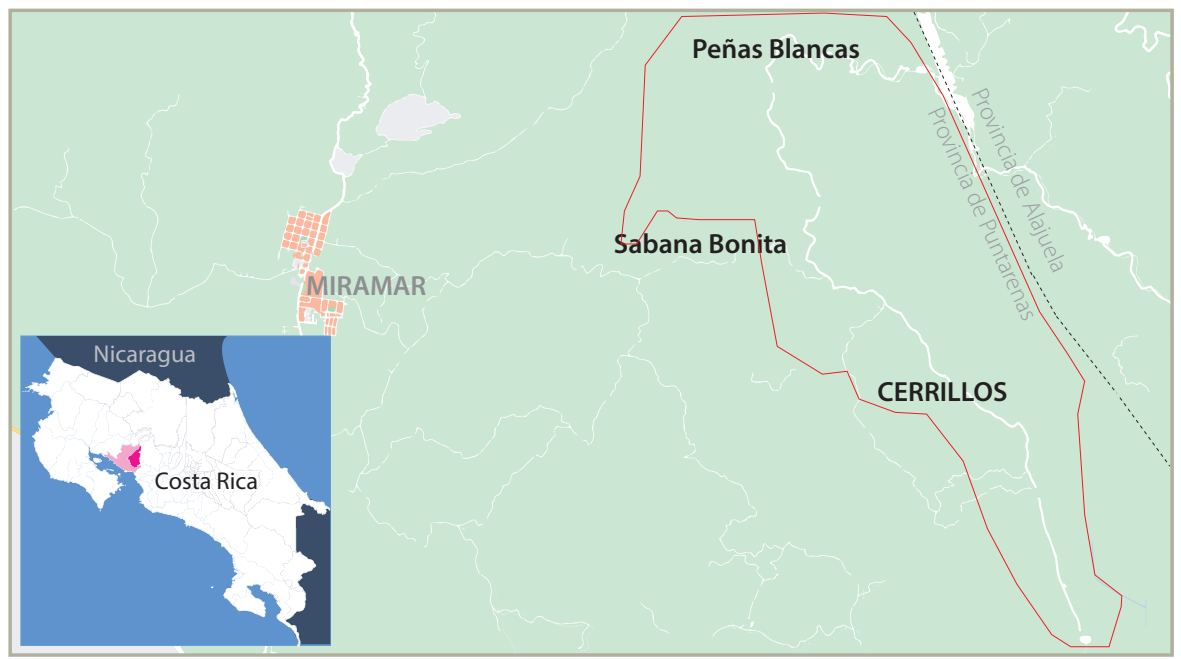

Fuente: Universidad de Costa Rica-Programa de Gestión Ambiental Integral (PROGAI 2007).

\section{Herramienta utilizada}

Los resultados que se muestran en este artículo son el efecto de la aplicación del MDRES diseñado por Chassoul (2015), específicamente el apartado al manejo de residuos a través de ecotecnologías. Como ecotecnologías adecuadas para la zona, se propone el uso de biodigestores, biojardineras y compost. Para esto se aplicó el proceso descrito en la figura 2.

Una vez conocida la situación sobre el manejo de los residuos en los poblados, el diseño e implementación de ecotecnologías fue asumido por la Asociación Pro Cuenca Río Jabonal en trabajo conjunto con la Sede de Occidente de la Universidad de Costa Rica. Esto ayudó a que las comunidades implementaran diversas ecotecnologías que les permitieron mejorar el manejo que brindan a los residuos. 
Figura 2

Proceso de aplicación del apartado de manejo de residuos del MDRES

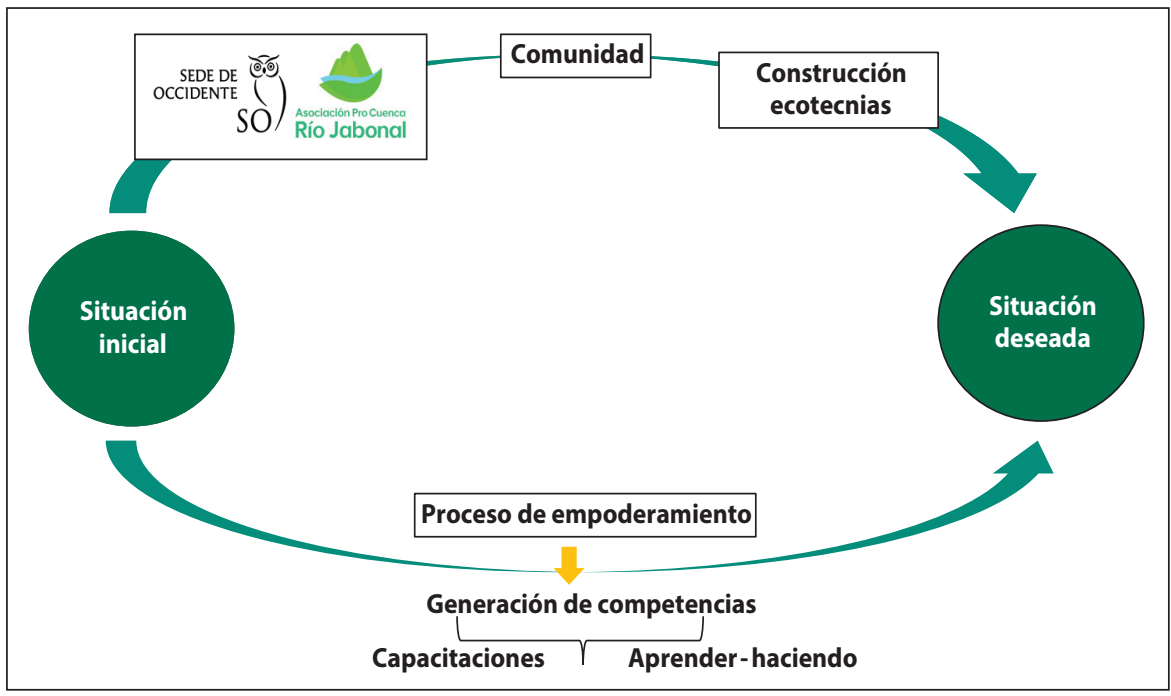

Elaboración propia.

Es importante señalar que, aunque muchas personas mostraron una disposición al cambio, o sea, manifestaron el deseo de implementar tecnologías para el manejo de residuos, en algunos casos no se materializaron debido a las barreras de control como la falta de oportunidades, habilidades y recursos (Charpentier 2004); por tanto, fue de vital importancia el empoderamiento de los habitantes, que incluyó la superación de barreras por medio del desarrollo de competencias a través de capacitaciones o el aprender-haciendo durante las construcciones.

\section{Metodología para el análisis de resultados}

Para medir los resultados de la implementación se utilizaron diversos indicadores divididos en temas como: energía, agua, residuos, producción y depuración de agua (ver detalle en la tabla 2). 
Se utilizó la prueba de proporciones con la finalidad de investigar si existían diferencias estadísticamente significativas entre el valor de los indicadores obtenidos en 2011, antes de la implementación de las ecotecnologías, y de 2015, después de la implementación. Esta prueba utilizó la estadística de chi cuadrado para efectuar el análisis (Walpone, Myers y Myers 1999).

En la prueba se plantearon dos hipótesis estadísticas:

H0: $\mathrm{p} 1=\mathrm{p} 2$

H1: $\mathrm{p} 1>\mathrm{p} 2$

Donde:

p1 = proporción obtenida en 2015

p2 = proporción obtenida en 2011

El nivel de significancia utilizado fue de $\alpha=0,05$. Cuando el $p$-valor es mayor que el nivel de significancia de la prueba, se falla en rechazar la hipótesis nula (); no se tiene evidencia suficiente para confirmar lo contrario. Si el p-valor es menor que el nivel de significancia, se falla en aceptar la hipótesis nula ().

\section{Resultados y análisis}

En total se construyeron 37 ecotecnologías, las cuales se detallan en la tabla 1 .

Tabla 1

Tipos de ecotecnologías construidas y cantidad en los poblados de Cerrillos, Sabana Bonita y Peñas Blancas

\begin{tabular}{|l|c|}
\hline \multicolumn{1}{|c|}{ Ecotecnología } & Cantidad \\
\hline Biojardineras & 8 \\
\hline Biodigestores & 5 \\
\hline Aboneras & 7 \\
\hline Microinvernaderos & 17 \\
\hline
\end{tabular}

Elaboración propia. 
Las competencias, para que los habitantes lograran el empoderamiento en este tema, se brindaron por medio de una serie de talleres como se muestra en la tabla 2.

Tabla 2

Competencias establecidas a nivel individual y de grupos productivos, actividades realizadas y participantes

\section{Competencias a nivel individual y organizacional (grupos productivos)}

Brinda un tratamiento adecuado a los residuos generados en las viviendas, las fincas y las microempresas utilizando ecotecnologías.

\begin{tabular}{|c|c|c|c|}
\hline Conocimientos & Actividades & Institución & Participantes \\
\hline \multirow[t]{3}{*}{$\begin{array}{l}\text { Sobre el funcionamien- } \\
\text { to y construcción de } \\
\text { ecotecnologías (biojar- } \\
\text { dineras, biodigestores, } \\
\text { producción de abono } \\
\text { orgánico) }\end{array}$} & $\begin{array}{l}\text { Taller práctico } \\
\text { de biodigestores. } \\
\text { Taller de operación y } \\
\text { mantenimiento } \\
\text { de biodigestores. }\end{array}$ & $\begin{array}{l}\text { Asociación Pro Cuen- } \\
\text { ca Río Jabonal } \\
\text { ICE }\end{array}$ & $\begin{array}{l}\text { Habitantes interesados de } \\
\text { Cerrillos, Peñas Blancas y } \\
\text { Sabana Bonita. } \\
\\
\text { Habitantes con biodiges- } \\
\text { tores de Cerrillos, Peñas } \\
\text { Blancas y Sabana Bonita. }\end{array}$ \\
\hline & $\begin{array}{l}\text { Taller práctico } \\
\text { de producción } \\
\text { de abono orgánico. }\end{array}$ & $\begin{array}{l}\text { Asociación Pro Cuen- } \\
\text { ca Río Jabonal }\end{array}$ & 4 familias. \\
\hline & $\begin{array}{l}\text { Taller práctico } \\
\text { de biojardineras. }\end{array}$ & $\begin{array}{l}\text { Asociación Pro Cuen- } \\
\text { ca Río Jabonal-UCR }\end{array}$ & 7 familias. \\
\hline $\begin{array}{l}\text { Sobre el concepto de } \\
\text { residuo y subproducto. } \\
\text { Sobre los tipos de } \\
\text { residuos sólidos y } \\
\text { líquidos. } \\
\text { Sobre la importancia } \\
\text { del manejo adecuado } \\
\text { de residuos. } \\
\text { Sobre técnicas para el } \\
\text { tratamiento y aprove- } \\
\text { chamiento de residuos. }\end{array}$ & $\begin{array}{l}\text { Taller teórico sobre } \\
\text { manejo de residuos. }\end{array}$ & UCR & $\begin{array}{l}\text { Habitantes de los pobla- } \\
\text { dos de Cerrillos, Sabana } \\
\text { Bonita y Peñas Blancas. } \\
\text { Grupo Mujeres Empren- } \\
\text { dedoras Peñas Blancas. }\end{array}$ \\
\hline
\end{tabular}

Fuente: Chassoul (2015). 
Los resultados obtenidos por tipo de ecotecnología construida se detallan a continuación:

Biojardineras: se construyeron 8 biojardineras para el tratamiento de aguas residuales de tipo ordinario provenientes de las viviendas y de la producción de queso. Como se observa en la figura 3, tratar el agua con la biojardinera permite cumplir con los parámetros establecidos por el art. 20 del Reglamento de Vertido y Reúso de Aguas Residuales (MINAE 2007), a excepción del DBO y las sustancias activas al azul de metileno. Sin embargo, esto sucede porque los valores utilizados de referencia son los de aguas residuales de tipo ordinario vertidas a un cuerpo receptor y dos de las biojardineras son utilizadas para el tratamiento de aguas residuales de tipo especial provenientes de la producción de queso. El Reglamento, en el art. 23, establece que para aguas provenientes de manufactura de productos lácteos el DBO debe ser menor o igual a $200 \mathrm{mg} / \mathrm{L}$, el DQO de $500 \mathrm{mg} / \mathrm{L}$ y los sólidos suspendidos totales de $100 \mathrm{mg} / \mathrm{L}$. Si se aplican estos valores, todos los parámetros quedan bajo lo establecido por el Reglamento.

Figura 3

\section{Comparación entre los valores obtenidos al final del tratamiento con los valores reglamentados}

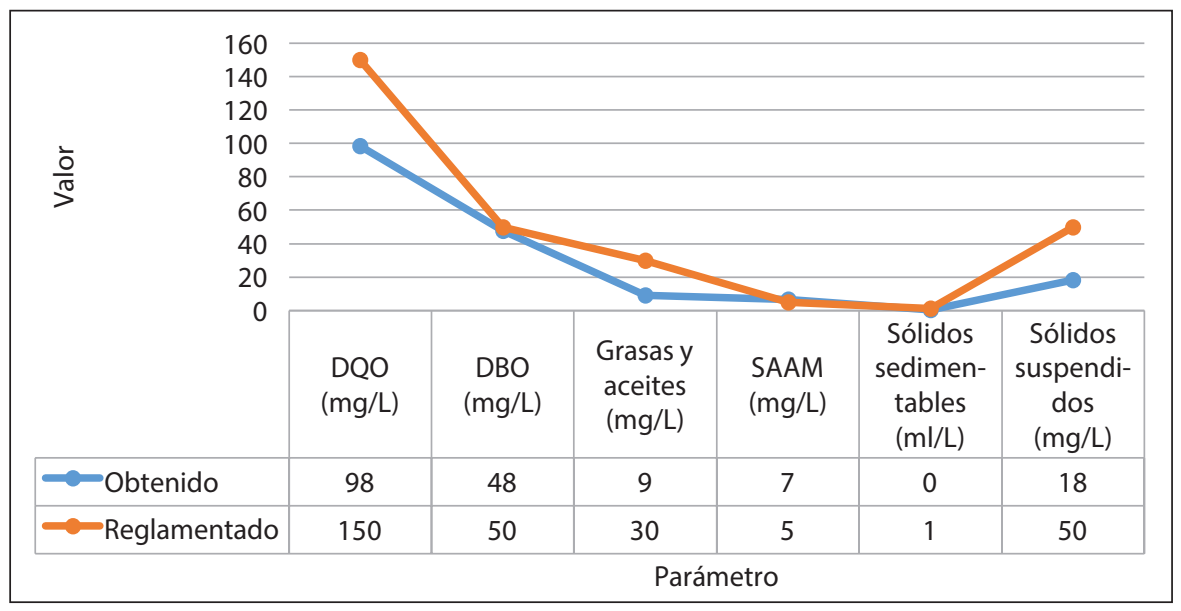

Elaboración propia. 
Sin embargo, es importante aclarar que este resultado es solo una muestra de lo que se puede obtener y no tiene un valor científico como tal, debido a que la cantidad de muestras tomadas en el año no son representativas, a causa de la limitación presupuestaria. Sin embargo, diversos estudios sobre biojardineras o humedales artificiales han demostrado efectividad en la reducción de la contaminación de aguas residuales de tipo ordinario (Romero-Aguilar et al. 2009; Quintero 2014; De la Cruz 2015; Úsuga et al. 2017; Asprilla, Ramírez y Rodríguez 2020).

Biodigestores: se construyeron cuatro biodigestores de bolsa plástica tubular de 5 metros de longitud y 2 metros de diámetro. Estos producen, aproximadamente, $172 \mathrm{~m}^{3} /$ año de biogás, lo cual permite a una familia de 3 o 4 personas cocinar los alimentos diarios.

Aboneras: se instalaron siete aboneras para brindar tratamiento a los residuos orgánicos en las casas por medio de lombrices. Se está produciendo compost para autoconsumo que utilizan en los jardines de las viviendas y en los microinvernaderos. La cantidad de compost aproximado que se produce por vivienda es de $410 \mathrm{~kg} / \mathrm{año}$, lo cual les brinda beneficios económicos, sociales y ambientales; económicos, al no tener que comprar otro tipo de abono para la producción de hortalizas y recibir ingresos por la posible venta de los mismos; sociales, al tener acceso a productos orgánicos, y ambientales, al no botar los residuos de las casas y utilizar abonos ecológicos.

Microinvernaderos para producción de hortalizas: se les brindó microinvernaderos para la producción casera de hortalizas a 17 familias, en los cuales se produce lechuga, culantro, espinaca, chile y tomate, principalmente. Las hortalizas obtenidas son principalmente para autoconsumo, pero algunas veces comparten con los vecinos o las venden.

Porta recipientes para separación de residuos: se construyeron e instalaron siete porta recipientes para la separación de residuos, como: vidrio, papel, cartón, aluminio, hojalata y plástico. Todos los residuos son recolectados por la municipalidad de Esparza. Con esto evitan que sean enterrados o vertidos en lotes baldíos.

Algunas de las tecnologías construidas se muestran en la figura 4. 
Figura 4

Ecotecnologías construidas en la subcuenca del río Jabonal (biodigestor [a], abonera [b], biojardinera [c] y microinvernadero [d])

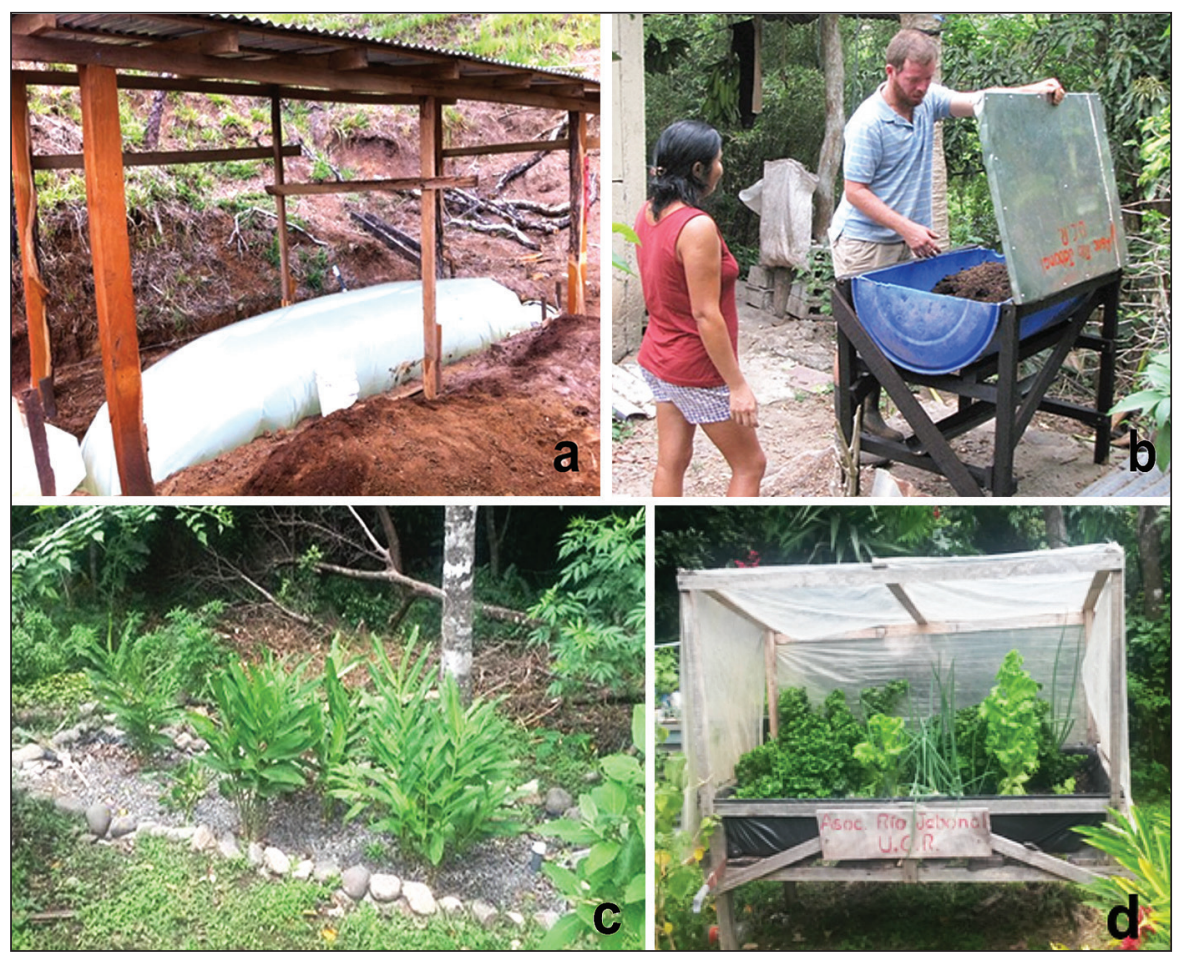

Elaboración propia.

El impacto de la aplicación del MDRES, específicamente en la construcción de ecotecnias en los habitantes de los tres poblados, se obtuvo por medio de la aplicación de los indicadores seleccionados. Los resultados se muestran en la tabla 3.

Con el propósito de investigar si existían diferencias estadísticamente significativas entre el valor de los indicadores obtenidos en 2011, antes de la implementación de las ecotecnologías, y el valor de 2015, después de la implementación, se aplicó la prueba de proporciones. 
Tabla 3

Indicadores establecidos y resultados obtenidos

\begin{tabular}{|c|c|c|c|c|}
\hline Tema & Subtema & Indicador & Definición & Resultado \\
\hline Energía & $\begin{array}{l}\text { Fuentes alternativas } \\
\text { de generación de } \\
\text { energía }\end{array}$ & $\begin{array}{l}\text { Utilización de fuentes alter- } \\
\text { nativas para la generación } \\
\text { de energía (viviendas con } \\
\text { biodigestores) }\end{array}$ & $\%$ del total & 7 \\
\hline \multirow[b]{2}{*}{ Agua } & \multirow{2}{*}{$\begin{array}{l}\text { Tratamiento de aguas } \\
\text { residuales }\end{array}$} & $\begin{array}{l}\text { Aguas residuales de tipo } \\
\text { ordinario tratadas }\end{array}$ & $\mathrm{m}^{3} / a n ̃ o$ & 1051,2 \\
\hline & & $\begin{array}{l}\text { Viviendas con tratamiento } \\
\text { de aguas residuales de tipo } \\
\text { ordinario }\end{array}$ & $\%$ del total & 11,3 \\
\hline \multirow{5}{*}{ Residuos } & \multirow{4}{*}{$\begin{array}{l}\text { Tratamiento residuos } \\
\text { sólidos }\end{array}$} & $\begin{array}{l}\text { Viviendas con tratamiento } \\
\text { de residuos orgánicos }\end{array}$ & $\%$ del total & 11,3 \\
\hline & & $\begin{array}{l}\text { Viviendas con separación } \\
\text { total de residuos }\end{array}$ & $\%$ del total & 11,3 \\
\hline & & Residuos tratados (boñiga) & kg/año & 38.210 \\
\hline & & $\begin{array}{l}\text { Residuos tratados (domésti- } \\
\text { cos biodegradables) }\end{array}$ & $\mathrm{kg} / \mathrm{año}$ & 4380 \\
\hline & $\begin{array}{l}\text { Capacitación en } \\
\text { manejo de residuos }\end{array}$ & $\begin{array}{l}\text { Habitantes capacitados en } \\
\text { manejo de residuos }\end{array}$ & $\%$ del total & 12,3 \\
\hline \multirow{3}{*}{ Producción } & Biogás & Cantidad de biogás & $\mathrm{m}^{3} /$ biodigestor/año & 171,6 \\
\hline & Lombricomposta & Cantidad de compost & $\mathrm{kg} / \mathrm{año}$ & 18.803 \\
\hline & Fertilizante orgánico & Cantidad fertilizante & Litros/biod/año & 17.520 \\
\hline \multirow{6}{*}{$\begin{array}{l}\text { Depuración } \\
\text { de agua }\end{array}$} & \multirow{6}{*}{$\begin{array}{l}\text { Aguas residuales de } \\
\text { tipo ordinario }\end{array}$} & DBO & \multirow{6}{*}{ \% disminución } & 81 \\
\hline & & DQO & & 74 \\
\hline & & Sólidos suspendidos & & 90 \\
\hline & & Grasas/aceites & & 74 \\
\hline & & Sólidos sedimentables & & 89 \\
\hline & & $\begin{array}{l}\text { Sustancias activas azul } \\
\text { metileno }\end{array}$ & & 48 \\
\hline
\end{tabular}

Fuente: adaptado de Chassoul (2015). 
Los resultados obtenidos se muestran en la tabla 4. Es importante aclarar que el análisis no se realizó a todos los indicadores, debido a que el resultado de algunos indicadores es consecuencia del cambio de otros, por lo que se escogieron los más representativos.

\section{Tabla 4}

Resultados del p-valor obtenido de la comparación de las proporciones del valor logrado en 2011 y 2015

\begin{tabular}{|l|c|c|c|c|}
\hline \multicolumn{1}{|c|}{ Indicador } & Definición & $\mathbf{2 0 1 1}$ & $\mathbf{2 0 1 5}$ & p-valor \\
\hline $\begin{array}{l}\text { Fuentes alternativas para la } \\
\text { generación de energía }\end{array}$ & $\%$ de viviendas & $2,8 \%$ & $7,0 \%$ & 0,122 \\
\hline $\begin{array}{l}\text { Viviendas con tratamiento de aguas } \\
\text { residuales de tipo ordinario }\end{array}$ & $\%$ de viviendas & $0 \%$ & $11,3 \%$ & 0,002 \\
\hline $\begin{array}{l}\text { Viviendas con tratamiento de } \\
\text { residuos orgánicos }\end{array}$ & $\%$ de viviendas & $1,41 \%$ & $11,3 \%$ & 0,008 \\
\hline $\begin{array}{l}\text { Viviendas con separación total de } \\
\text { residuos }\end{array}$ & $\%$ de viviendas & $0 \%$ & $11,3 \%$ & 0,002 \\
\hline
\end{tabular}

Fuente: adaptado de Chassoul (2015).

Como se observa, el único indicador que no es significativamente diferentes es el porcentaje de viviendas con fuentes alternativas de generación de energía. En 2015, el 7 \% de las viviendas (5) utilizaban biodigestores para la generación de gas para cocina, y en 2011 correspondía al 2,8\% (2). Este cambio no es una diferencia estadísticamente significativa, pero el incremento del uso de biodigestores proporcionó un beneficio económico a las familias que lo poseen, ahorrándoles en promedio USD 200 dólares por año a cada familia. Los biodigestores generan aproximadamente $171,6 \mathrm{~m}^{3}$ de gas por biodigestor por año y procesan $17.520 \mathrm{~kg}$ de boñiga por año. Entre el uso de la boñiga para la producción de compost y la generación de gas, se procesaron $38.210 \mathrm{~kg}$ de boñiga en un año, lo cual permitió reducir la emisión de metano al aire.

La cantidad de casas que cuentan con biojardineras para el tratamiento de aguas residuales de tipo ordinario sí es estadísticamente significativo. Al 
inicio del proyecto en ninguna vivienda se trataban las aguas residuales y al final de la implementación del modelo, ocho viviendas contaban con biojardineras. Esto ha permitido que las aguas residuales vertidas cumplan con los parámetros establecidos por el Reglamento de Vertido y Reúso de Aguas Residuales (MINAE 2007).

La diferencia entre la cantidad de viviendas que cuentan con un sistema de tratamiento de residuos orgánicos y con separación total de residuos, es estadísticamente significativa. Este valor pasó de 1 a 8 viviendas, en el caso de tratamiento de residuos orgánicos y de 0 a 8 , en el caso de la separación total de residuos reciclables. El contar con un sistema de tratamiento de residuos orgánicos ha permito en un año transformar aproximadamente 4380 $\mathrm{kg}$ de residuos a $3285 \mathrm{~kg}$ de abono orgánico, que es utilizado en los jardines de las viviendas.

Tal como se muestra en los resultados, el proyecto fue exitoso, sin embargo, es valedero hacerse la pregunta de cómo está la situación en 2020. Se puede responder que sobre algunas ecotecnias se logró el empoderamiento de los habitantes, por ejemplo, las ocho biojardineras siguen funcionando y las siete aboneras y los 17 microinvernaderos siguen produciendo. De hecho, estos dos últimos se han replicado en pueblos vecinos o han aumentado en tamaño. Sin embargo, de los cinco biodigestores solo uno se encuentra en uso, debido a daños en la bolsa ocasionados, en la mayoría de los casos, por animales y a pesar de que las familias conocen los aportes económicos que estos les brindan, no cuentan con el presupuesto para comprar una bolsa nueva e instalarla o consideran que el mantenimiento les requiere de mucho tiempo.

\section{Conclusiones}

Con los resultados obtenidos, se demostró que el uso de las ecotecnologías implementadas contribuye a disminuir el impacto negativo que generan las acciones de los habitantes en el ambiente y a la vez permite comprobar que desde las viviendas y las fincas se pueden desarrollar proyectos ambiental y económicamente viables. Sin embargo, la adopción de ecotecnologías por los habitantes es un proceso lento y se requiere contar con un grupo que 
lidere la implementación, como es en este caso la Asociación Pro Cuenca Río Jabonal. Cuando hay convencimiento de la utilidad y efectividad del uso de ecotecnologías se da una extensión de los conocimientos a otros miembros de la comunidad.

Además de la aportación positiva que realizan estas ecotecnologías al ambiente, su uso favorece otros ámbitos familiares. Por ejemplo, el uso de biodigestores y microinvernaderos favorecen el presupuesto familiar. La utilización de biojardineras permite tratar las aguas residuales de tipo ordinario de las viviendas y las aguas residuales de tipo especial de las queseras y cumplir con los parámetros establecidos por ley, de una forma más económica y agradable a la vista. El cultivo de hortalizas en los microinvernaderos provee productos frescos y libres de químicos y favorece la seguridad alimentaria de las familias. Sin embargo, es importante retomar el tema de los biodigestores y realizar un análisis, en conjunto con los beneficiarios de esta tecnología, para determinar las causas de lo sucedido y entender qué factores provocaron que no fueran exitosos, con la finalidad de evitar que se repitan los mismos errores en futuros proyectos.

El aprender-haciendo demostró ser una metodología válida y efectiva para la capacitación de adultos y junto con los talleres permitió el aprendizaje de todos los miembros que participaron en los proyectos.

Por otra parte, vale mencionar la importancia de la supervisión y seguimiento a las ecotecnologías implementadas para que sigan funcionando en el tiempo, papel que cumple la Asociación, quienes han tenido que disminuir sus actividades por limitaciones económicas; por lo que sería conveniente que la Universidad siga brindando apoyo a través de proyectos, para que la Asociación pueda buscar fuentes de financiamiento alternativas y el esfuerzo realizado no se pierda.

\section{Referencias}

Asprilla, Wilson, Johan Ramírez y Diana Catalina Rodríguez. 2020. "Humedales artificiales subsuperficiales: comparación de metodologías de diseño para el cálculo del área superficial basado en la remoción de la materia orgánica". Ingenierías USBMed 11 (1): 65-73. https://doi.org/10.21500/20275846.4558. 
Brix, Hans, y Carlos Arias. 2006. Historia y generalidades en el uso de humedales para el control de la contaminación acuática. Aarhus: Aarhus University.

CABAL. 2014. Guía de buenas prácticas en proyectos de biodigestores. Managua: Cabal. https://bit.ly/384sLW7.

Charpentier, Claudia. 2004. "Las barreras de la educación ambiental pueden superarse". Revista Biocenosis 18 (1-2): 103-108. https://bit.ly/2NWPIUr.

Chassoul, María José. 2015. "Modelo de desarrollo sostenible para las comunidades de Sabana Bonita, Cerrillos y Peñas Blancas, Esparza, Puntarenas, Costa Rica”. Tesis doctoral, Instituto Tecnológico de Costa Rica / Universidad Nacional / Universidad Estatal a Distancia, San José. https://bit.ly/3cj9ZM3.

Chassoul, María José, y Claudia Charpentier. 2017. "Modelo de desarrollo sostenible para tres comunidades en Costa Rica". Revista de Ciencias Ambientales 52 (2): 171-183. https:// doi.org/10.15359/rca.52-2.10.

Comisión Nacional para el Desarrollo de los Pueblos Indígenas (CDI). 2016. Ecotecnias. Guía práctica para comunidades indígenas. Accedido septiembre de 2020. https://bit. ly/3uL8WNi.

Cubillo, María Fernanda, y William Gómez. 2017. "Biojardineras como alternativas para el tratamiento de aguas residuales: experiencia en cinco biojardineras en las comunidades de Barra Honda y La Vigía de Nicoya, Guanacaste". Revista Universidad en Diálogo 7 (1): 69-87. http://dx.doi.org/10.15359/udre.7-1.4.

De la Cruz, Blanca. 2015. "Evaluación de los servicios ecosistémicos proporcionados por los humedales artificiales. Aplicación al humedal artificial de Carrícola (Valencia)”. Tesis de grado, Universidad Politécnica de Valencia, Valencia. http://hdl.handle.net/10251/54587.

Environmental Protection Agency (EPA). 1993. Subsurface Flow Constructed Wetland for Wastewater Treatment. Washington D. C.: EPA.

CR Ministerio de Ambiente y Energía (MINAE). 2007. Reglamento de Vertido y Reúso de Aguas Residuales. San José. La Gaceta 55, 19 de marzo.

Quintero, Jesús. 2014. "Evaluación de humedales artificiales pilotos de flujo horizontal y tipo superficial y subsuperficial para el tratamiento de aguas residuales”. Ingenium. Revista de la Facultad de Ingeniería 15 (29): 85-112. https://doi.org/10.21500/01247492.1347.

Ríos, Ney, Aura Cárdenas, Hernán Andrade, Muhammad Ibrahim, Francisco Jiménez, Freddy Sancho, Elías Ramírez, Bismark Reyes y Alfredo Woo. 2006. "Escorrentía superficial e infiltración en sistemas ganaderos convencionales y silvopastoriles en el trópico subhúmedo de Nicaragua y Costa Rica". Agroforestería en las Américas 45: 66-71. https://bit. ly/3uPpJi6.

Rivas, Olga, Margie Faith y Rossy Guillén. 2009. "Biodigestores: factores químicos, físicos y biológicos relacionados con su productividad”. Tecnología en Marcha 23 (1): 39-46. https://revistas.tec.ac.cr/index.php/tec marcha/article/view/132.

Romero-Aguilar, Mariana, Arturo Colín-Cruz, Enrique Sánchez-Salina y Laura Ortiz-Hernández. 2009. "Tratamiento de aguas residuales por un sistema piloto de humedales arti- 
ficiales: evaluación de la remoción de la carga orgánica". Revista Internacional de Contaminación Ambiental 25 (3): 157-167. http://www.scielo.org.mx/pdf/rica/v25n3/v25n3a4. pdf.

Universidad de Costa Rica / Programa de Gestión Ambiental Integral (PROGAI). 2007. Gestión participativa de la subcuenca del río Jabonal. Informe de proyecto de investigación. San José: PROGAI.

Universidad Nacional de Ingeniería (UNI). 2004. Aplicación de la tecnología de biofiltros como una alternativa viable para el tratamiento de aguas residuales domésticas en países de clima tropical. San José: UNI.

Úsuga, Fabián, Andrés Patiño, Diana Rodríguez y Gustavo Peñuela. 2017. "Kinetic Study and Removal of Contaminants in the Leachate Treatment Using Subsurface Wetlands at Pilot Scale". Revista ION 30 (2): 55-63. http://dx.doi.org/10.18273/revion.v30n2-2017005.

Valdez, Bladimir. 2016. "Evaluación de la calidad de biogás y biol producido en biodigestores usando excretas de animales del parque zoológico de Huachipa". Tesis de grado, Universidad Agraria La Molina, Lima. https://bit.ly/3bV2P09.

Venegas, José Apolonio, Anastasio Espejel Pérez, José Alberto Castellanos y Gaudencio Sedano. 2017. "Potencial de energía eléctrica y factibilidad financiera para biodigestor-moto generador en granjas porcinas de Puebla". Revista Mexicana de Ciencias Agrícolas 8 (3): 735-740. https://doi.org/10.29312/remexca.v8i3.47.

Walpone, Ronald, Raymond Myers y Sharon Myers. 1999. Probabilidad y estadística para ingenieros. Ciudad de México: Prentice-Hall Hispanoamérica.

Zhang, Dongqing, Richard Gersberg y Tan Soon Keat. 2009. "Constructed Wetlands in China". Ecological Engineering 35: 1367-1378. https://bit.ly/2OdcLtR. 